PROCEEDINGS OF THE

AMERICAN MATHEMATICAL SOCIETY

Volume 125, Number 8, August 1997, Pages 2443-2451

S 0002-9939(97)03870-7

\title{
KEEPING ADDITIVITY OF THE NULL IDEAL SMALL
}

\author{
JINDŘICH ZAPLETAL \\ (Communicated by Andreas R. Blass)
}

\begin{abstract}
We shall show that various statements are consistent with additivity of the null ideal equal to $\aleph_{1}$; for example, "all branchless trees of size $\aleph_{1}$ are special", (S) conjecture and "there are only five cofinal types of directed posets of size $\aleph_{1} "$.
\end{abstract}

\section{INTRODUCTION}

In this paper we provide machinery for proving that a certain large class of forcings has a certain regularity property. The class in question includes posets used for

(1) specializing branchless trees $[\mathrm{S}]$,

(2) (S) conjecture [T2],

(3) embedding the poset for adding $\aleph_{1}$ Cohen reals into a given poset of uniform density $\aleph_{1}[\mathrm{SZ}]$,

(4) classification of directed posets [T1] or transitive relations [T3] of size $\aleph_{1}$,

(5) other "side condition" combinatorics on $\aleph_{1}$; e.g. shooting an uncountable set through a coherent sequence on $\omega_{1}$ [T2].

The regularity property we obtain implies preservation of additivity of the ideal of Lebesgue null sets. As a corollary, it is consistent with ZFC set theory that additivity of the null ideal is $\aleph_{1}$ and all statements obtainable through (1)-(5) above hold, that is, all branchless trees of size $\aleph_{1}$ are special, (S) conjecture holds etc. Thus a definite limitation on a canonical variation of the powerful "side condition" method has been exacted for the first time.

Our notation follows the set-theoretical standard as set forth in $[\mathrm{J}]$. In a forcing notion, $p \leq q$ means " $p$ is more informative than $q$ ". A tree $T$ of height $\omega_{1}$ is special if there is a function $f: T \rightarrow \omega$ with $s<t$ in $T$ implying $f(s) \neq f(t)$. Trees grow upwards. (S) conjecture is the statement "every hereditarily separable Hausdorff space is hereditarily Lindelöf". The symbol $\mathcal{N}$ denotes the collection of Borel null sets, often confused with their Borel codes. $H_{\kappa}$ is the set of all sets of hereditary cardinality $<\kappa$. If $N$ is an elementary submodel of $H_{\kappa}$ and $P \in N$ is a forcing, a condition $p \in P$ is called $N$-master if for every dense set $D \subset P$ in $N$, the set $D \cap N$ is predense below $p$.

Received by the editors November 8, 1995 and, in revised form, February 26, 1996.

1991 Mathematics Subject Classification. Primary 03E35, 03E50.

Research at MSRI partially supported by NSF grant \# DMS 9022140. The author wishes to thank Itay Neeman for asking the original inspiring question. 


\section{LOCALIZATION}

Definition 1. Let $\mathfrak{F} \subset{ }^{\omega} \omega$ and let $e$ be a positive integer. The family $\mathfrak{F}$ is said to be $e$-localized if there exists a function $h: \omega \rightarrow[\omega]<\aleph_{0}$ such that

(1) $|h(n)| \leq n^{e}$ for every integer $n$,

(2) for every $f \in \mathfrak{F}$ there is an integer $n \in \omega$ so that for every $m>n, f(m) \in h(m)$ holds.

If $e=1$ then the family $\mathfrak{F}$ is said to be localized.

The relevance of the above definition is revealed in the result of Bartoszyński [B, BJ Section 2.3.A] saying that for a transitive model $M$ of ZFC the following are equivalent:

(1) $M \cap{ }^{\omega} \omega$ is localized;

(2) $M \cap{ }^{\omega} \omega$ is $e$-localized for some positive integer $e$;

(3) the union of all Lebesgue measure zero Borel sets coded in $M$ has measure zero.

There is a natural c.c.c. forcing for making the set of ground model reals localized $[\mathrm{Tr}]$ and there are some preservation theorems for "the set of ground model reals is not localized" [JS], [BJ]. We shall prove that a large class of forcings preserves unlocalized families in a strong sense.

Definition 2. (1) [JS] Let $\kappa$ be a large regular cardinal and $N \prec H_{\kappa}$. We say that a function $f \in{ }^{\omega} \omega$ is $N$-big if for every $h: \omega \rightarrow[\omega]<\aleph^{0}$ in the model $N$ with $|h(n)| \leq n$ the set $\{m \in \omega: f(m) \notin h(m)\}$ is infinite.

(2) Let $P$ be a forcing. We say that $P$ is friendly if for every $p \in P$, every large enough regular cardinal $\kappa$, every countable elementary submodel $N \prec H_{\kappa}$ with $p, P$ in $N$ and every $N$-big function $f \in \omega^{\omega} \omega$ there is an $N$-master condition $q \leq p$ such that $q \Vdash$ " $f$ is $N[G]$-big".

The important point is that it is possible to iterate friendly forcings preserving the statement "the family of ground model reals is not localized" or equivalently, "U $(\mathcal{N} \cap V) \notin \mathcal{N} "$-Lemma 13. Obviously, a finite iteration of friendly forcings is friendly and friendliness is inherited by regular subposets. In [JS] it is proved that the random algebra as well as every $\sigma$-centered forcing is friendly. We considerably extend these results.

\section{Specializing trees}

The purpose of this section is to prove that the usual specializing forcing for a branchless tree of height $\omega_{1}$ is friendly. The technique will be of great use in the next section. For now, fix a tree $T$ of height $\omega_{1}$ and no branches of length $\omega_{1}$. There is no restriction on the size of levels of $T$.

Definition 3. (1) If $a, b$ are disjoint finite subsets of $T$ then we say that $a$ and $b$ fit together if for every $s \in a$ and $t \in b, s$ and $t$ are incompatible as elements of $T$.

(2) The specialization forcing is $P=\{p: p$ is a finite function from $T$ to $\omega$ such that $s<_{T} t$ in $\operatorname{dom}(p)$ implies $\left.p(s) \neq p(t)\right\}$ ordered by reverse inclusion.

Lemma 4. Let $\left\{a_{\alpha}: \alpha \in \omega_{1}\right\}$ be a family of pairwise disjoint finite subsets of $T$. Then there is an infinite set $Y \subset \omega_{1}$ such that $a_{\alpha}: \alpha \in Y$ pairwise fit together. 
Proof. The usual proof of c.c.c.-ness of $P$ [S, p. 103] shows that there are $\alpha \neq \beta$ with $a_{\alpha}, a_{\beta}$ fitting together. The lemma follows from the Erdős-Dushnik-Miller partition relation $\omega_{1} \rightarrow\left(\omega_{1}, \omega\right)$ applied to the function $f:\left[\omega_{1}\right]^{2} \rightarrow 2$ defined by $f(\alpha, \beta)=0$ iff $a_{\alpha}, a_{\beta}$ fit together.

Now assume that $\kappa$ is a large regular cardinal, $N \prec H_{\kappa}$ is a countable submodel with $T \in N$ and $f$ is $N$-big. We shall prove that $P \Vdash$ " $f$ is $N[G]$-big". Then, since the forcing $P$ is c.c.c., any condition in it is $N$-master and it witnesses the friendliness of $P$ as desired.

For contradiction, let $p \in P, n \in \omega$ and $\dot{h} \in N$ be such that

(1) $p \Vdash$ "for all $m \in \omega,|\dot{h}(m)| \leq m$ ",

(2) $p \Vdash$ "for every $m>n, \check{f}(m) \in \dot{h}(m)$ ".

Let $p_{0}=p \cap N \in N$. By c.c.c.-ness of $P$, by strengthening the condition $p$ if necessary one can arrange that $p_{0} \Vdash$ "for all $m \in \omega,|\dot{h}(m)| \leq m$ ".

Work in $N$. Fix an integer $m>n$ and by a tree induction construct a tree $X \subset{ }^{<\omega}(\omega+1)$, a partition $X=X_{0} \cup X_{1}$ and a function $F: X \rightarrow P$ so that

(1) the empty sequence 0 is in $X$ and $F(0)=p_{0}$,

(2) $s \subset t$ in $X$ implies $F(s) \geq F(t)$ in $P$,

and for each $s \in X$ with $l t h(s)=i$ exactly one of the following holds:

(3) either, there is a sequence $\left\langle q_{j}: j \in \omega\right\rangle$ such that each $q_{j} \leq F(s)$ forces in $P$ that $i \in \dot{h}(m)$, and moreover, the sets $\operatorname{dom}\left(q_{j} \backslash F(s)\right): j \in \omega$ pairwise fit together. In this case, $s \in X_{0}$, the set of successors of $s$ in $X$ is exactly $\left\{s^{\wedge}\langle j\rangle: j \in \omega\right\}$ and $F\left(s^{\wedge}\langle j\rangle\right)=q_{j}$,

(4) or, no such sequence exists. Then $s \in X_{1}$, the only successor of $s$ in the tree $X$ is $s^{\curvearrowright}\langle\omega\rangle$ and $F\left(s^{\curvearrowright}\langle\omega\rangle\right)=F(s)$.

A set $o(X) \subset \omega$ is defined by $i \in o(X)$ iff there exists a function $G: X \rightarrow \omega$ such that for every sequence $s \in X$ of length $i$, if $\forall j \in i s(j)>G\left(s\lceil j)\right.$ then $s \in X_{0}$.

Claim 5. $|o(X)| \leq m$.

Proof. Suppose for a contradiction that there are $m+1$ elements of $|o(X)|$, enumerated in the increasing order as $i_{0}$ through $i_{m}$. Pick witnesses $G_{k}: k \leq m$ for $i_{k} \in o(X)$. Then for every sequence $s \in X$ of length $i_{m}+1$ such that $\forall j \leq i_{m} \forall k \leq$ $m s(j)>G_{k}(s\lceil j)$ (and there are plenty of these) the value $F(s)$ as an element of $P$ forces each one of the $m+1$ distinct integers $i_{k}: k \leq m$ into the set $\dot{h}(m)$. But this is absurd, since $p \geq F(s)$ and $p \Vdash|\dot{h}(m)| \leq m$.

Claim 6. $f(m) \in o(X)$.

Proof. The proof of this fact takes place outside of the model $N$. To define the witness $G: X \rightarrow \omega$ for $f(m) \in o(X)$, consider two cases:

(1) $s \in X_{1}$. Then let $G(s)=0$.

(2) $s \in X_{0}$. By (3) above, $\left\{a_{j}=\operatorname{dom}\left(F\left(s^{\frown}\langle j\rangle\right) \backslash F(s)\right): j \in \omega\right\}$ is a family of pairwise disjoint fitting finite subsets of the tree $T$. There is an integer $j_{0}$ such that for every $j>j_{0}$, the sets $a_{j}$ and $\operatorname{dom}\left(p \backslash p_{0}\right)$ fit together; set $G(s)=j_{0}$.

The existence of an integer $j_{0}$ as in (2) above can be demonstrated as follows. By elementarity of the model $N$, if $u \in N \cap T$ and $t \in \operatorname{dom}\left(p \backslash p_{0}\right)$ are compatible as elements of the tree $T$, then necessarily $u<_{T} t$, for if $t<_{T} u$ then $t \in N$, contradicting the definition of the condition $p_{0}$. By the mutual fitting of the $a_{j}$ 's, only finitely many of the sets $a_{j} \subset N \cap T$ can have nonempty intersection with the 
finitely many linearly ordered subsets $\left\{u \in N \cap T: u<_{T} t\right\}: t \in \operatorname{dom}\left(p \backslash p_{0}\right)$ of the tree $T$. Any $j_{0}$ larger than the indexes of these sets will do.

Why does the function $G$ have the desired properties? Well, choose an arbitrary sequence $s \in X$ of length $f(m)$ with $\forall j \in f(m) s(j)>G(s \uparrow j)$. It is necessary to verify that $s \in X_{0}$. By the definition of $G$ and $X$, the condition $F(s)$ is compatible with $p$. By induction on $\alpha \in \omega_{1}$ construct a sequence $\left\langle a_{\alpha}: \alpha \in \omega_{1}\right\rangle$ so that

(1) $a_{\alpha}$ are finite pairwise disjoint subsets of the tree $T$,

(2) for every $\alpha \in \omega_{1}$ there is $q \leq F(s)$ such that $q \Vdash$ " $f(m) \in \dot{h}(m)$ " and $a_{\alpha}=$ $\operatorname{dom}(q \backslash F(s))$.

Note that any sequence $\left\langle a_{\alpha}: \alpha \in \beta\right\rangle$ of countable length $\beta$ satisfying $(1,2)$ above for $\alpha \in \beta$ can be prolonged further to some $\left\langle a_{\alpha}: \alpha \in \beta+1\right\rangle$ such that $(1,2)$ continue to hold. For if this were not the case, by elementarity there would be a witness $\left\langle a_{\alpha}: \alpha \in \beta\right\rangle \in N$ which cannot be prolonged. However, such a sequence from the model $N$ can be prolonged using $a_{\beta}=\operatorname{dom}\left(p \backslash p_{0}\right)$, with the condition $q=F(s) \cup p$ witnessing the property (2) at $\beta$.

By elementarity of the model $N$, there is a sequence $\left\langle a_{\alpha}: \alpha \in \omega_{1}\right\rangle$ satisfying $(1,2)$ above in $N$. It follows from Lemma 4 that the case (3) of definition of the tree $X$ is valid at $s$ and $s \in X_{0}$.

Now within the model $N$, for each integer $m>n$ construct a tree $X_{m}$ and a set $o\left(X_{m}\right)$ as above. The function $g: \omega \backslash n \rightarrow[\omega]^{<\aleph_{0}}$ defined by $g(m)=o\left(X_{m}\right)$ is in the model $N$ and contradicts the assumption of $f$ being $N$-big. Therefore, we have proved

Theorem 6. Let $T$ be a tree of height $\omega_{1}$ without branches of height $\omega_{1}$. Then the standard T-specialization forcing is friendly.

Remark. The result becomes rather trivial if the tree $T$ is supposed to have countable levels. In such a case, it is easy to prove that every real added by the specialization forcing comes from a Cohen-generic extension. Thus the specialization forcing for $T$ must necessarily be friendly by results of [JS].

Remark. The same technology can be used to demonstrate friendliness of a number of finite condition forcings, whose c.c.c. is proved in a certain canonical manner. For example, let $\left\langle f_{\alpha}: \alpha \in \omega_{1}\right\rangle \subset{ }^{\omega} \omega$ be a modulo finite increasing unbounded sequence of increasing functions. Let the partition $H:\left[\omega_{1}\right]^{2} \rightarrow 2$ be defined as $H(\alpha, \beta)=0$ if $\alpha<\beta$ and there is an integer $n \in \omega$ with $f_{\alpha}(n)>f_{\beta}(n)$. It is known [T2] that the poset $P$ of finite 0 -homogeneous sets ordered by reverse inclusion is c.c.c. and destroys the unboundedness of the sequence. Using the same method as above, it is possible to show that $P$ is friendly. The following is open:

Question 7. Is OCA [T2] consistent with additivity of the null ideal equal to $\aleph_{1}$ ?

\section{SidE CONDITION FORCINGS}

The purpose of this section is to define the class of ideal-based forcings and to prove friendliness of elements of this class. Our scheme is designed to comprehend many side condition forcings as used in the work of S. Todorcevic [T1], [T2], [T3] and others. Let $A$ be a set of finite subsets of $\omega_{1}$ ordered by $\sqsubseteq$ and let $\mathfrak{I}$ be an ideal on $\omega_{1}$ such that the following axioms are satisfied:

(A) $\sqsubseteq$ refines the inclusion, for each $a \in A$ and $\beta \in \omega_{1} a \cap \beta \sqsubseteq a$ holds and if $a, b$ are both in $A$ and $\sqsubseteq$-compatible then $a \cup b \in A$ is their $\sqsubseteq$-upper bound. 
(B) I contains singletons, every $\mathfrak{I}$-positive set has a countable $\mathfrak{I}$-positive subset and the $\sigma$-ideal $\sigma \mathfrak{I}$ generated by $\mathfrak{I}$ is proper.

Moreover, for each $a \in A$ there are

(C) a $\sigma \mathfrak{I}$ positive set $Z \subset \omega_{1}$ such that $a \sqsubseteq a \cup\{\beta\} \in A$ holds for every $\beta \in Z$,

(D) an I-large set $Y \subset \omega_{1}$ such that for every $\beta \in Y$ the implication $a \cap \beta \sqsubseteq$ $(a \cap \beta) \cup\{\beta\} \in A \rightarrow a \sqsubseteq a \cup\{\beta\} \in A$ holds.

The pair $\langle A, \sqsubseteq\rangle$ is to be understood as a problematic finite-condition forcing construction for which c.c.c. cannot be proved, or which collapses $\aleph_{1}$ outright. The existence of the ideal ensures that there is a way to add a $\sqsubseteq$-filter which meets many dense subsets of $\langle A, \sqsubseteq\rangle$. Fix a large regular cardinal $\kappa$. The ideal-based forcing $P$ derived from $A, \sqsubseteq, \Im$ has the following form:

$P=\left\{f: f\right.$ is a finite function from $\omega_{1}$ to $H_{\kappa}$, for $\alpha \in \operatorname{dom}(f) f(\alpha)=\left\langle M_{\alpha}, \xi_{\alpha}\right\rangle$ and

(E) the set $\operatorname{body}(f)=\left\{\xi_{\alpha}: \alpha \in \operatorname{dom}(f)\right\}$ is in $A$,

(F) every $M_{\alpha}$ is a countable elementary submodel of $H_{\kappa}$ containing $A$, $, \mathfrak{I}, f\lceil\alpha$,

(G) $\left.\xi_{\alpha} \notin \bigcup\left(M_{\alpha} \cap \mathfrak{I}\right)\right\}$.

The order on $P$ is defined by $f \leq g$ if $g \subset f$ and $\operatorname{body}(g) \sqsubseteq \operatorname{body}(f)$.

The forcing $P$ adds a $\sqsubseteq$-filter $\{a \in A: a=b o d y(p)$ for some $p \in G\}$ which meets all dense subsets of $\langle A, \sqsubseteq\rangle$ which are in some sense large as measured by $\mathfrak{I}$.

Frequently, for the sake of preservation of $\aleph_{2}$ one needs to consider an amended variation of $P$ which has matrices of models as side conditions instead of just an $\in$-chain of models as above [T2]. We call such forcings amended ideal-based; since our proofs carry over to the class of amended ideal-based forcings with only more complicated notation, we concentrate on the class of ideal-based forcings proper. The point of course is that this class is reasonably wide; indeed, our scheme includes many of the side condition posets used in the literature. The following fact provides a by no means complete list.

Fact 8. The forcings for the following problems are (amended) ideal-based:

(1) (S) conjecture [T2],

(2) making a poset of uniform density $\aleph_{1}$ add $\aleph_{1}$ Cohen reals [SZ],

(3) classification of transitive relations on $\aleph_{1}$ [T1], [T3],

(4) shooting an uncountable set through a coherent sequence on $\omega_{1}$ [T2].

Proof. We consider the case of (S) conjecture. As in [T2], it is only necessary to cope with the following problem. Let $\omega_{1}$ be equipped with topology $\mathfrak{T}$ so that the space $\left(\omega_{1}, \mathfrak{T}\right)$

(1) is hereditarily separable, that is, for every $X \subset \omega_{1}$ there is a countable subset $Y \subset X$ with the same closure,

(2) is not hereditarily Lindelöf, and it is even right separated, that is, for each $\alpha \in \omega_{1}$ there is an open set $O_{\alpha}$ such that $\alpha \in O_{\alpha}$ and the closure of $O_{\alpha}$ is a subset of $\alpha+1$.

We wish to violate the hereditary separability of the space $\left(\omega_{1}, \mathfrak{T}\right)$ by introducing an uncountable discrete subset to it. The forcing for doing that [T2] can be cast as an ideal-based forcing derived from $A=\left[\omega_{1}\right]^{<\kappa_{0}}, a \sqsubseteq b$ just in case $a \subset b$ and for every $\xi \in(b \backslash a)$ and every $\zeta \in a \xi \notin O_{\zeta}$; furthermore, $\mathfrak{I}=\left\{X \subset \omega_{1}\right.$ : the closure of $X$ is countable $\}$. It is not difficult to check the axioms (A) through (D) in the definition of ideal-based. (B) follows from hereditary separability and (D) from $\mathfrak{I}$-smallness of every $O_{\alpha}$. 
The intended uncountable discrete set will be $\bigcup\{\operatorname{body}(f): f \in G\}$, where $G \subset P$ is a generic filter.

Theorem 9. Any ideal-based forcing $P$ is proper and friendly.

Proof. Let $A$, $, \mathfrak{I}, \kappa$ be the parameters from which $P$ is defined. To prove the properness, let $p_{0} \in P, \lambda$ be a large regular cardinal, let $N \prec H_{\lambda}$ be a countable elementary submodel with $p_{0}, A \sqsubseteq, \mathfrak{I}, \kappa \in N$, and let $\delta=N \cap \omega_{1}$. By (C) there is a countable ordinal $\xi$ such that $\xi \notin \bigcup(\mathfrak{I} \cap N)$ and $\operatorname{body}\left(p_{0}\right) \sqsubseteq \operatorname{body}\left(p_{0}\right) \cup\{\xi\} \in A$. Let $p_{1}=p_{0} \cup\left\{\left\langle\delta,\left\langle N \cap H_{\kappa}, \xi\right\rangle\right\rangle\right\}$. Obviously, $p_{1} \leq p_{0}$ and we shall show that $p_{1}$ is a master condition for the model $N$. Thus, for any dense set $D$ of $P$ which happens to be in $N$, the set $D \cap N$ must be proved predense below $p_{1}$. Fix $p_{2} \leq p_{1}$ and a dense set $D \in N$; we shall produce conditions $p_{5} \leq p_{2}$ and $q \in D \cap N$ with $p_{5} \leq q$, completing the proof of properness. By strengthening $p_{2}$ if necessary, it can be assumed that there is an element of $D$ above $p_{2}$.

Let $p_{3}=p_{2} \cap N$. Obviously $p_{3} \in P \cap N$ and $p_{2} \leq p_{3}$, by (A). The whole point of the proof is to find a way of carefully extending $p_{3}$ within $N$ while preserving compatibility with $p_{2}$. Let $k=\left|p_{2} \backslash p_{3}\right|$ and $\xi_{0} \ldots \xi_{k-1}$ enumerate $\operatorname{body}\left(p_{2}\right) \backslash \operatorname{body}\left(p_{3}\right)$ in the increasing order. By induction on $l<k$ define sets $S(t)(l) \subset \omega_{1}$ for all $t \in{ }^{<\omega} \omega_{1}$ simultaneously by

(1) $S(t)(0)=\left\{\zeta \in \omega_{1} \backslash \max \left(\operatorname{body}\left(p_{3}\right) \cup r n g(t)\right): \exists p_{4} \leq p_{3}\right.$ such that $p_{4}$ has an element of $D$ above it and $\operatorname{body}\left(p_{3}\right) \cup r n g(t) \sqsubseteq \operatorname{bod} y\left(p_{4}\right)=\operatorname{bod} y\left(p_{3}\right) \cup r n g(t) \cup$ $\{\zeta\} \in A\}$.

(2) $S(t)(l+1)=\left\{\zeta \in \omega_{1} \backslash \max \left(\operatorname{body}\left(p_{3}\right) \cup r n g(t)\right): \operatorname{body}\left(p_{3}\right) \cup r n g(t) \sqsubseteq \operatorname{body}\left(p_{3}\right) \cup\right.$ $r n g(t) \cup\{\zeta\} \in A$ and the set $S\left(t^{\frown}\langle\zeta\rangle\right)(l)$ is $\mathfrak{I}$-positive $\}$.

Claim 10. The set $S(\langle\rangle)(k-1)$ is $\mathfrak{I}$-positive.

Proof. Note that the system $\left\{S(t)(l): t \in{ }^{<\omega} \omega_{1}, l<k\right\}$ belongs to all the models mentioned in $p_{2}$ above $\delta$ since it is in $N \cap H_{\aleph_{2}}$. The claim will be proved by contradiction. If $S(\langle\rangle)(k-1)$ were an element of $\mathfrak{I}$, by induction on $l<k$ one could show that $\xi_{l} \notin S\left(\left\langle\xi_{l^{\prime}}: l^{\prime}<l\right\rangle\right)(k-1-l)$. But this is a contradiction to the case $(1)$ of the definition of the system $\left\{S(t)(l): t \in{ }^{<\omega} \omega_{1}, l<k\right\}$, since $\xi_{k-1} \in S\left(\left\langle\xi_{l}: l<k-1\right\rangle\right)(0)$ as witnessed by the condition $p_{2}$.

Now by induction on $l<k$ build $\zeta_{l}, T_{l}, X_{l}$ so that

(1) for $l \leq k, a_{l}=\left\langle\zeta_{l^{\prime}}: l^{\prime}<l\right\rangle$ is an increasing sequence of countable ordinals larger than $\max \left(\operatorname{bod} y\left(p_{3}\right)\right)$ in $N$,

(2) $T_{l} \in N$ is an $\mathfrak{I}$-positive countable subset of the $\mathfrak{I}$-positive set $S\left(a_{l}\right)(k-l-1)$, by $(\mathrm{B})$,

(3) $\operatorname{body}\left(p_{2}\right)$ and $\operatorname{body}\left(p_{3}\right) \cup r n g\left(a_{l}\right)$ are $\sqsubseteq$-compatible and an $\mathfrak{I}$-large set $X_{l} \subset \omega_{1}$ is a witness to (D) for $\operatorname{body}\left(p_{2}\right) \cup r n g\left(a_{l}\right)$,

(4) $\zeta_{l} \in T_{l} \cap X_{l}$.

By the construction, $a_{k} \in N$, $\operatorname{body}\left(p_{2}\right)$ and $\operatorname{body}\left(p_{3}\right) \cup r n g\left(a_{k}\right)$ are $\sqsubseteq$-compatible and moreover, there is a condition $p_{4} \leq p_{3}$ such that there is an element of $D$ above it and $\operatorname{bod} y\left(p_{4}\right)=\operatorname{body}\left(p_{3}\right) \cup r n g\left(a_{k}\right)$. By the elementarity of the model $N$, there are such $p_{4}$ and $q \in D$ above it already in $N$. By the definition of the forcing $P$, $p_{5}=p_{4} \cup p_{2}$ is a lower bound of $p_{4}$ and $p_{2}$ and has $q \in D \cap N$ above it as desired.

The friendliness of $P$ is proved by a trick similar to the one in Section 2. Let us adopt the framework from the proof of properness of $P$, in particular, choose 
$p_{0}, N \ldots$ and the master condition $p_{1} \leq p_{0}$ for the model $N$ as constructed above. Let $f \in{ }^{\omega} \omega$ be an $N$-big function. We shall show that $p_{1} \Vdash$ " $f$ is $N[G]$-big".

For contradiction, let $p_{2} \leq p_{1}, \dot{h} \in N$ and $n \in \omega$ be such that

(1) $p_{2} \Vdash$ "for all integers $m,|\dot{h}(m)| \leq m$ ",

(2) $p_{2} \Vdash$ "for all integers $m>n, \check{f}(m) \in \dot{h}(m)$ ".

Let $p_{3}=p_{2} \cap N$. Then $p_{3} \geq p_{2}$ is in $N$ and by strengthening the condition $p_{2}$ if necessary we may assume that $p_{3} \Vdash$ "for all integers $m,|\dot{h}(m)| \leq m$ ". Let $k=\left|p_{2} \backslash p_{3}\right| \geq 1$.

Work in $N$. Fix an integer $m>n$. By a tree induction construct a tree $X \subset{ }^{<\omega} \omega$, its subset $X_{0}$ and functions $F: X \rightarrow P, T: X_{0} \rightarrow V$ so that:

(1) the empty sequence 0 is in $X$ and $F(0)=p_{3}$,

(2) for every $s \subset t$ both in $X, F(t) \leq F(s)$ holds in $P$.

Moreover, at each $s \in X$, exactly one of the two following cases will hold.

Case 1. There is a tree $T(s)$ on $\leq k \omega_{1}$ such that

(a) the empty sequence is in $T(s)$ and $T$ consists of increasing sequences of ordinals above $\max (\operatorname{body}(F(s)))$,

(b) for every $t \in T(s)$ of length $<k$ the set $\left\{\zeta \in \omega_{1}: t^{\curvearrowright}\langle\zeta\rangle \in T(s)\right\}$ is countable and $\mathfrak{I}$-positive; moreover for each $\zeta$ in this set, body $(F(s)) \cup \operatorname{rng}(t) \sqsubseteq$ $\operatorname{body}(F(s)) \cup \operatorname{rng}(t) \cup\{\zeta\} \in A$,

(c) for every $t \in T(s)$ of length $k$ (i.e. a terminal node) there is a condition $q_{t} \leq F(s)$ in $P$ such that $\operatorname{bod} y\left(q_{t}\right)=\operatorname{body}(F(s)) \cup r n g(t)$ and $q_{t} \Vdash$ "length $(s) \in$ $\dot{h}(m) "$.

In this case, let $s \in X_{0}, T(s)$ will be the value of $T$ at $s$ and $s^{\frown}\langle l\rangle \in X$ for all $l \in \omega$. Also, $F\left(s^{\wedge}\langle l\rangle\right): n \in \omega$ enumerates the set $\left\{q_{t}: t \in T(s)\right.$ is a terminal node $\}$.

Case 2. No such tree exists. Then $s \notin X_{0}$, the only successor of the sequence $s$ in $X$ is $s^{\wedge}\langle 0\rangle$ and $F(s)=F\left(s^{\frown}\langle 0\rangle\right)$.

This completes the inductive definition of the tree $X$ in $N$. Define a set $o(X) \subset \omega$ by $i \in o(X)$ if there exists a collection $\left\{A(s, t): s \in X_{0}, t \in T(s)\right.$ is not a terminal node $\}$ of $\Im$-large sets so that for each sequence $u \in X$ of length $i$ if $\left(^{*}\right)$ below holds for all $j<i$ then $u \in X_{0}$.

$\left.{ }^{*}\right)$ If $u\left\lceil j \in X_{0}\right.$ then $F\left(u\lceil(j+1))=q_{t}\right.$ for some terminal node $t \in T(u\lceil j)$ such that $\forall l<k t(l+1) \in A(u\lceil j, t\lceil l)$.

Claim 11. $|o(X)| \leq m$.

Proof. For contradiction, suppose that there are $m+1$ elements of $o(X)$ enumerated in the increasing order as $i_{0}$ through $i_{m}$. Pick witnesses $\left\{A_{l}(s, t): s \in X_{0}, t \in T(s)\right.$ is not a terminal node $\}$ for $i_{l} \in o(X)$ and define $\left\{A(s, t): s \in X_{0}, t \in T(s)\right.$ is not a terminal node $\}$ by $A(s, t)=\bigcap_{l \leq m} A_{l}(s, t)$. Now each $A(s, t)$ is still an $\mathfrak{I}$ large set and so it is possible to find a sequence $u \in X$ of length $i_{m}+1$ such that $\left(^{*}\right)$ holds for each $j<l t h(u)$. But then, from the construction of $X$ and $F$ it follows that $F(u) \Vdash$ " $\left\{i_{0} \ldots i_{m}\right\} \subset \dot{h}(m)$ ", which is absurd since $F(u) \leq p_{3}$ and $p_{3} \Vdash$

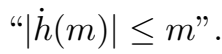

Claim 12. $f(m) \in o(X)$.

Proof. It is necessary to define the witness collection; our candidate lies outside of $N$. For $s \in X_{0}$ and $t \in T(s)$ there will be two cases: 
(1) Either $\operatorname{body}\left(p_{2}\right)$ and $\operatorname{body}(F(s)) \cup r n g(t)$ are $\sqsubseteq$-compatible. In such a case let $A(s, t)$ be an I-large witness to (D) for $\operatorname{body}\left(p_{2}\right) \cup \operatorname{body}(F(s)) \cup r n g(t)$.

(2) Otherwise let $A(s, t)=\omega_{1}$.

Now suppose that a sequence $u \in X$ of length $f(m)$ satisfies $(*)$ for all $j<f(m)$. By the definition of $A(s, t)$ and the tree $X$, necessarily body $(F(u))$ and body $\left(p_{2}\right)$ are $\sqsubseteq$-compatible and therefore $p_{2}$ and $F(u)$ are compatible conditions in $P$. The proof of $u \in X_{0}$ is essentially a repetition of the proof of the properness of $P$ with the condition $p_{2}$ replaced with $F(u) \cup p_{2}$ and the phrase "element of $D$ above it" replaced with "forces $f(m)$ into $\dot{h}(m)$ ".

Now the definition of $X, o(X)$ was uniform for integers $m>n$. Thus within the model $N$ there is a sequence $X_{m}, o\left(X_{m}\right): m>n$ such that $\left|o\left(X_{m}\right)\right| \leq m$ and $f(m) \in o\left(X_{m}\right)$ for every $m>n$. But then the sequence $o\left(X_{m}\right): m>n$ in the model $N$, understood as a function of $m$, contradicts $N$-bigness of the function $f$.

It should be remarked that while ideal-based forcings preserve $\operatorname{add}(\mathcal{N})$, they can add dominating functions. If $\left\langle f_{\alpha}: \alpha \in \omega_{1}\right\rangle \subset{ }^{\omega} \omega$ is a modulo finite increasing unbounded sequence of increasing functions then it is possible to derive an S-space from it [T2]. Then the ideal-based forcing killing that space adds a function which modulo finite dominates all $f_{\alpha}: \alpha \in \omega_{1}$.

\section{Conclusion}

At last, we are in a position to construct some interesting models of set theory with the additivity of the null ideal equal to $\aleph_{1}$. The classical iteration vehicle gives

Lemma 13. Let $\left\langle P_{\alpha}: \alpha \leq \theta, \dot{Q}_{\alpha}: \alpha<\theta\right\rangle$ be a countable support iteration of forcings such that $P_{\alpha} \Vdash$ " $\dot{Q}_{\alpha}$ is friendly" for each $\alpha<\theta$. Then $P_{\theta} \Vdash$ "the union of the null sets coded in the ground model is not null".

It seems likely that in fact $P_{\theta}$ is a friendly forcing, but we have no argument for that.

Proof. By induction on $\beta \leq \theta$ we shall demonstrate that $P_{\beta} \Vdash$ "the union of the null sets coded in the ground model is not null". The limit step is handled by [BJ, Theorem 6.3.41]. For the successor step, assume that $P_{\beta} \Vdash$ "the union of the null sets coded in the ground model is not null"; we shall prove the same statement for $P_{\beta+1}$. Choose a generic filter $G \subset P_{\beta}$ and work in $V[G]$. It is enough to show that $Q_{\beta} \Vdash$ " $V \cap{ }^{\omega} \omega$ is not localized". For contradiction, suppose $q \in Q_{\beta}, \dot{h}$ are such that $q \Vdash_{Q_{\beta}}$ "for all $n \in \omega,|\dot{h}(n)| \leq n$ and $\dot{h}$ localizes $V \cap{ }^{\omega} \omega$ ". Choose a large regular cardinal $\kappa$ and a countable elementary submodel $N \prec H_{\kappa}$ with $q, Q_{\beta}, \dot{h} \in N$.

Claim 14. There is an $N$-big function $f \in V \cap{ }^{\omega} \omega$.

Proof. By an easy bookkeping argument, there is a function $k: \omega \rightarrow[\omega]<\aleph_{0}$ such that $\forall n \in \omega|k(n)| \leq n^{2}$ and for every function $l \in N$ with $l: \omega \rightarrow[\omega]^{<\aleph_{0}}$, $\forall n \in \omega|l(n)| \leq n$ there is an integer $m_{0}$ such that for every $m>m_{0}, l(m) \subset k(m)$. By the induction hypothesis, $V \cap{ }^{\omega} \omega$ is not 2-localized and therefore there is a function $f \in V \cap \cap^{\omega} \omega$ such that the set $\{m \in \omega: f(m) \notin k(m)\}$ is infinite. Obviously, the function $f$ is $N$-big.

The postulated friendly master condition $r \leq q$ for $N, f$ contradicts the assumption $q \Vdash$ " $\exists n \forall m>n f(m) \in \dot{h}(m)$ ". 
Therefore, starting from a model of the Continuum Hypothesis, any sufficiently generic iteration of length $\omega_{2}$ of proper, $\aleph_{2}$-p.i.c. [S, pg. 262] and friendly forcings will provide for $\Vdash^{*} a d d(\mathcal{N})=\aleph_{1}, \mathfrak{c}=\aleph_{2}$, all branchless trees of size $\aleph_{1}$ are special, (S) conjecture holds, every poset of uniform density $\aleph_{1}$ adds $\aleph_{1}$ Cohen reals etc." In the construction, it is necessary to use amended ideal-based forcings in order to ensure $\aleph_{2}$-c.c. of the resulting iteration. The standard bookkeeping arguments are left to the reader.

\section{REFERENCES}

[B] T. Bartosziński, Additivity of measure implies additivity for category, Trans. Amer. Math. Soc. 281 (1984), 209-213. MR 85b:03083

[BJ] T. Bartoszyński and H. Judah, Set theory. On the structure of the real line, A K Peters, Wellesley, Massachusets, 1995. MR 96k:03002

[J] T. Jech, Set Theory, Academic Press, New York, 1978. MR 80a:03062

[JS] H. Judah and S. Shelah, The Kunen-Miller chart, Jour. Symbolic Logic 55 (1990), 909-927. MR 91g:03097

[RS] J. Raisonnier and J. Stern, The strength of measurability hypothesis, Israel Jour. Math. 50 (1985), 337-349. MR 87d:03129

[Tr] J. Truss, Connection between different amoeba algebras, Fund. Math. 130 (1988), 137-155. MR 90h:03036

[S] S. Shelah, Proper Forcing, Springer-Verlag, New York, 1982. MR 84h:03002

[SZ] S. Shelah and J. Zapletal, Embeddings of Cohen algebras, Adv. Math. (to appear).

[T1] S. Todorcevic, Directed sets and cofinal types, Trans. Amer. Math. Soc. 290 (1985), 711-723. MR 87a:03084

[T2] — Partition problems in topology, Amer. Math. Soc., Providence, 1989. MR 90d:04001

[T3] - A classification of transitive relations on $\omega_{1}$, preprint, 1995.

M. S. R. I., 1000 Centennial Drive, Berkeley, California 94720

E-mail address: jindra@msri.org

Current address: Mailcode 253-37, California Institute of Technology, Pasadena, California 91125

E-mail address: jindra@cco.caltech.edu 